\title{
ANALYSIS OF A NEAR FIELD MIMO WIRELESS CHANNEL USING 5.6 GHZ DIPOLE ANTENNAS
}

\author{
Mohamed Ismaeel Maricar $^{(1)}$, Gabriele Gradoni ${ }^{(2)}$, Steve Greedy ${ }^{(1)}$, Michel T Ivrlac ${ }^{(3)}$, Josef A. Nossek ${ }^{(3)}$, \\ Sendy Phang ${ }^{(2)}$, Stephen C Creagh ${ }^{(2)}$, Gregor Tanner ${ }^{(2)}$ and Dave W P Thomas ${ }^{(1)}$ \\ ${ }^{1}$ : Department of Electrical and Electronics Engineering, University of Nottingham, NG7 2RD. \\ ${ }^{2}$ : School of Mathematical Sciences, University of Nottingham, NG7 2R3. \\ ${ }^{3}:$ Institute for Circuit Theory and Signal Processing, Technische Universität München,Munich Germany.
}

\begin{abstract}
Understanding the impact of interference upon the performance of a multiple input multiple output (MIMO) based device is of paramount importance in ensuring a design is both resilient and robust. In this work the effect of element-element interference in the creation of multiple channels of a wireless link approaching the near-field regime is studied. The elements of the 2-antenna transmit- and receive-arrays are chosen to be identical folded dipole antennas operating at $5.6 \mathrm{GHz}$. We find that two equally strong channels can be created even if the antennas interact at sub-wavelength distances, thus confirming previous theoretical predictions.
\end{abstract}

\section{INTRODUCTION:}

Modern chip structures and printed circuit boards (PCB) make use of multiple copper interconnects to distribute signals to different components. This may give rise to interference problems such as crosstalk and radiated emissions that are of broad interest in electromagnetic compatibility (EMC)[1]. As both the data rate and the integration levels increase, each metallic part of the chip/PCB driven by time domain currents becomes an efficient antenna at higher frequencies, thus jeopardizing signal integrity and energy efficiency [2]. Even though novel design strategies have been proposed to overcome EMC issues at PCB level, these will eventually reach their limits. Therefore, it becomes interesting to consider using radio-frequency (RF) interconnects. We have recently proposed [3] that this technology can be based on large-scale wireless communications, where high data rates can be achieved in the presence of interference from multipath propagation. In such a context, high gain and broadband on-chip/PCB antennas can be used to exploit and control radiated emissions in order to create fast and robust 3D wireless interconnects. This paradigm shift may pave the way towards a wireless on-chip or chipto-chip (c2c) technology that offers viable alternatives to wired interconnects and can thus overcome the limitations of copper conductors.

In this paper, we study the channel efficiency of a link established between two antenna arrays in the transition from near- to far-field. The arrays are identical and made of two folded dipole antennas that are fed individually. The robustness of communication between multiple-input multiple-output (MIMO) channels is investigated in presence of inter-element interference. The selected frequency of operation covers bands currently used in wireless local area networks (WLAN) as well as those considered for current and next generation mobile networks (4G LTE and 5G). Whilst the simulated microwave wireless link is well suited for a device-to-device communication, it will also serve as a model for understanding radiation from on-chip/PCB interconnects, where antennas and links need to be scaled down as the frequency is increased by at least a factor of 10. Numerical predictions are relevant for near-field measurements of radiated emission from small-scale MIMO wireless systems, as well as for the EMC modelling of wireless data transmission in presence of multiple interacting antennas.

\section{DESIGN OF A DIPOLE ANTENNA:}

The chosen antenna design was based on the dual band folded dipole configuration as presented [4] and figure 1 shows the schematic view of the dipole antenna. The dipole antenna has a metallization thickness (T) and the apex corner of the antenna is directly fed by a $50 \Omega$ microstrip line with the width (W), height of (h). Beveling $(\alpha)$ were introduced to increase the bandwidth and energy transmission of the dipole antennas. The complete antenna, is made up from two different dipoles on either side of the feed line and, in order to realize the physical size, the arms of the dipole antennas were effectively folded to extend the current path. The resulting dimensions of the antenna length and width were based on calculations obtained from [4]. The dipole antennas were analysed using Momentum, a planar electromagnetic simulation tool which is part of the ADS suite of design tools which utilizes the method of moments [5]. The dipole antennas were analyzed on FR4 material with a relative permittivity of $\varepsilon_{\mathrm{r}}=4.4$ and thickness of $1.4 \mathrm{~mm}$. The length and width of the $50 \Omega$ microstrip line were calculated by using the "line calculator" component of the ADS suite and it was found to be $2 \times 13.6 \mathrm{~mm}$. A number of simulations were then performed in order to find out the optimal resonant frequency and bandwidth of the antenna configuration. 


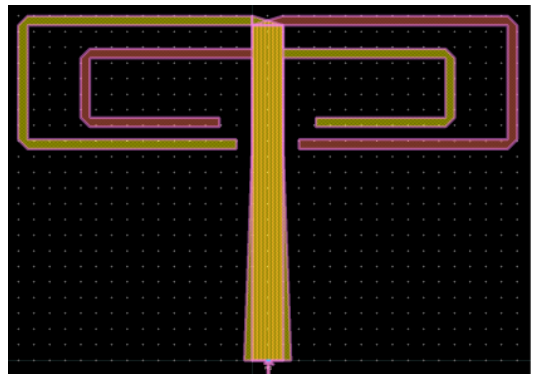

Figure 1 Schematic view of the dipole antenna.

\subsection{Simulation results of dipole antennas:}

Whilst using the simulation tool it was found that the choice of mesh size was particularly important in order to obtain a realistic return loss of the dipole antenna especially at higher frequencies [6]. Initial simulation results show that the dipole antennas are resonant at 5.6 $\mathrm{GHz}$ with a bandwidth of $\sim 1.2 \mathrm{GHz}$. The simulated frequency response of the input reflection co-efficient of the antenna is shown in Fig-2 (a) and it shows that the return loss of the dipole antennas is $\angle-10 \mathrm{~dB}$ over the frequency range of $4.2 \mathrm{GHz}$ to $6 \mathrm{GHz}$. 3D radiation pattern of the dipole antennas are simulated by using the MoM in ADS. Fig-2(b) shows the radiation pattern of the half wave dipole antennas in free space and it has gain of $3.5324 \mathrm{dBi}$.

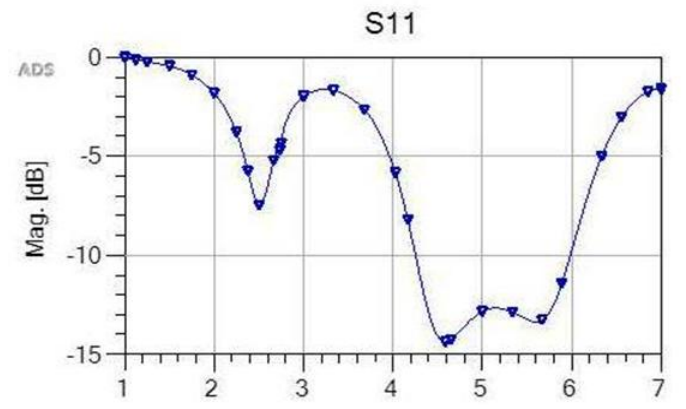

Figure 2 (a) Return loss of the dipole antenna.

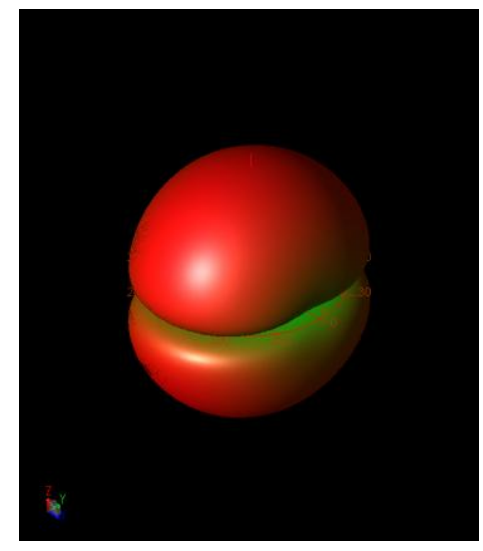

Fig 2 (b) Radiation Pattern of the dipole antennas

\section{MULTIPORT SYSTEM MODEL:}

The antenna is now used within a $2 \times 2$ MIMO configuration and its performance is analysed, again using Momentum. A schematic sketch of the antenna configuration within the larger system is depicted in Figure 3. The 4-antenna-system is modelled as a linear 4-port system, where 2 ports are on the Tx side and the remaining 2 ports on the $\mathrm{Rx}$ side. The results are described in the form of a scattering or impedance matrix [7], [8]. The channel strengths are then investigated in order to study the strongest channel between the $\mathrm{Tx}$ and $\mathrm{Rx}$ ends. Figure 4 shows the simulated results for the ratio $\left(\mathrm{s}_{1}{ }^{2} / \mathrm{s}_{2}{ }^{2}\right)$ of the power received at ports 3 and 4 over the bandwidth of a dipole. Here, $\mathrm{s}_{1}{ }^{2}$ and $\mathrm{s}_{2}{ }^{2}$ are the effective sub-channel power gains at ports 3 and 4 respectively. It is seen that if the separation of the elements in the Tx and Rx dipole pairs is $d=0.18 \lambda$ (see Figure 3), then for a $\operatorname{Rx}-\mathrm{Tx}$ array separation distance (D) with $\mathrm{D}=0.373 \lambda$ then we observe that $s_{1}=s_{2}$. This means that two equally strong channels can be set up between the Tx and Rx in the near field.

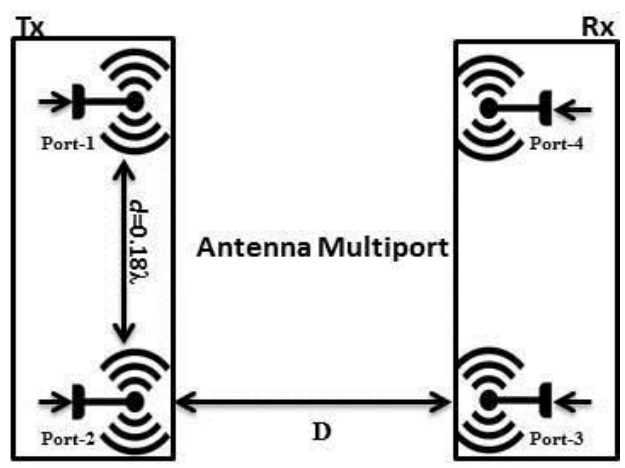

Figure 3 Near Field MIMO channel

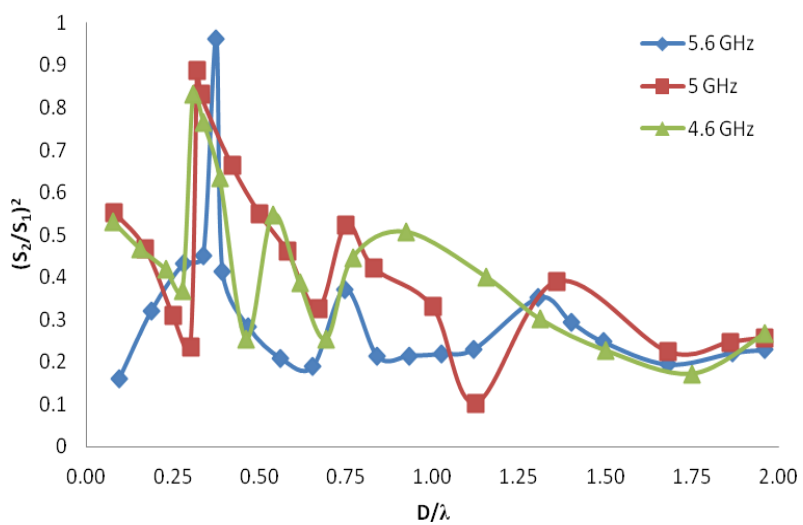

Figure 4 Two Strong channels MIMO system over the bandwidth 


\section{CONCLUSION:}

Interference between dipole antennas forming MIMO wireless channels were studied in the near field region. The results shows that if the separation between the dipoles in the Tx and Rx elements is $0.18 \lambda$, then for a Rx-Tx pair separation distance $\mathrm{D}=0.373 \lambda$, two the equally strong channel can be set up between the Tx and $\mathrm{Rx}$ in the near field.

\section{REFERENCE:}

[1] P. P. Pande and S. Vangal, "Guest Editors' Introduction: Promises and Challenges of Novel Interconnect Technologies," IEEE Des. Test Comput., vol. 27, no. 4, pp. 6-9, Jul. 2010.

[2] A. Alexiou and M. Haardt, "Smart antenna technologies for future wireless systems: trends and challenges," IEEE Commun. Mag., vol. 42, no. 9, pp. 90-97, Sep. 2004.

[3] D. G. G. and P. D. T. Dr. Gregor Tanner, Dr. Stephen Creagh, "Noisy electromagnetic fields a technological platform for chip-to-chip communication in the 21 st century," 2015.
[Online].Available:http://horizon2020projects.c om/es-future-emerging-technologies/h2020supports-nottingham-universitys-c2c-project/.

[4] X. W. D. and Q. W. Y.Y. Gua, X.M Zhang, G.L. Nig, D.Zhao, "Minizturized Modified Dipoles Antenna for WLAN Applications," Electromagentic Res. Lett., vol. 24, pp. 139147, 2011.

[5] Agilent Technologies, "Advanced Design System (ADS-2015)," 2015.

[6] M. I. Maricar, J. Glover, G. Evans, D. Cumming, and C. Oxley, "Design and characterization of a novel diamond resonator," Microw. Opt. Technol. Lett., vol. 56, no. 7, pp. 1691-1693, Jul. 2014.

[7] M. T. Ivrlac and J. A. Nossek, "The Multiport Communication Theory," IEEE Circuits Syst. Mag., vol. 14, no. 3, pp. 27-44, Jan. 2014.

[8] M. T. Ivrlač and J. A. Nossek, "Toward a Circuit Theory of Communication," IEEE Trans. Circuits Syst. I Regul. Pap., vol. 57, no. 7, pp. 1663-1683, Jul. 2010. 